\title{
Slow release anti-fungal skin formulations based on citric acid intercalated layered double hydroxides nanohybrids
}

\author{
Jayoda Perera', Manjula Weerasekera ${ }^{3}$ and Nilwala Kottegoda ${ }^{1,2^{*}}$
}

\begin{abstract}
Background: During the past few decades, the occurrence of superficial fungal infections has rapidly increased. As the fungal infections take longer time to get cured, concepts such as designing drugs with extended persistence and controlled release have gained attention. In this context, nanotechnology has been identified as the latest technological revolution which has opened up new pathways for designing new therapeutic materials. Out of the many available nano-structures layered double hydroxides have gained increased scientific attention in applications as slow and controlled release drug formulations. This study focuses on the encapsulation of citric acid which has anti-fungal properties into a Mg-Al- layered double hydroxide $(\mathrm{LDH})$ in order to be used as slow release topical skin formulations.
\end{abstract}

Results: Citrate ions were encapsulated into Mg-Al LDH using one step co-precipitation reaction. The successful intercalation of citrate ions into the layered structure has been proved referring to the expansion in the interlayer spacing as observed by the shift in the basal peak of the powder X-ray diffraction pattern. Fourier transform infra-red spectroscopy data suggests the change in the electron density around the carboxylate groups of the citrate ion thus providing evidences for formation of encapsulated hybrid composite. The resulting nanohybrid has been then, introduced into a general body cream formulation containing cocoa-butter. Both citrate LDH and the resulting body cream formulations demonstrated prolonged slow release characteristics up to $8 \mathrm{~h}$ in aqueous medium under different pH values $(3,4$, and 5) compared to quick and fast release of pure citric acid. It was observed that the slow reelase was most efficient at low pH values. The encapsulation between the nano-layers and citrate ions are the key to the slow release characteristics. The body cream has been tested for the anti-fungal activity against three common Candida species (C. albicans, C. glabrata, C. tropicalis). The novel nanohybrid has shown an improved activity and slow release characteristics up to $48 \mathrm{~h}$ against the $C$. albicans and $\mathrm{C}$. glabrata but not for $\mathrm{C}$. tropicalis.

Conclusion: The study confirms that the citrate ion intercalated LDHs have the potential for use in future slow release antifungal drug formulation.

Keywords: Layered double hydroxides, Citric acid, Nanohybrid, Anti-fungal, Candida species, Slow release

\section{Background}

Candida species are the most common cause of fungal infections [1]. Candida species produce diseases that range from non-life-threatening superficial to systemic infections that may involve virtually any organ [2]. As it can cause broad range of infections it requires a broad range of therapeutic strategies.

\footnotetext{
* Correspondence: nilwala@sjp.ac.lk

${ }^{1}$ Department of Chemistry, Faculty of Applied Science, University of Sri Jayewardenepura, Nugegoda, Sri Lanka

${ }^{2}$ Sri Lanka Institute of Nanotechnology, Center for Excellence in Nanotechnology, Nanotechnology and Science Park, Pitipana, Homagama, Sri Lanka

Full list of author information is available at the end of the article
}

Over the past few decades the word 'nano' has become rapidly insinuating into the world consciousness. It has already conjured up speculation about a seismic shift in almost every aspect of science and engineering including the advancements in medical sciences. This technology involves the use of particles or materials that have at least one dimension in the range of 1-100 nm size scale. In this size scale, unexpected properties of the material are derived by the resulted high surface area and the quantum mechanical effects. As a result, both chemical and physical properties of these materials are much more different compared to the bulk material. 
Nanotechnology has already shown potential in pharmaceutical industry [3]. It can be helpful from developing antibiotic drug to more wider and complex applications such as use of DNA - nanoparticle hybrid to store genetic information [4]. This technology becomes important in novel pharmaceutical approaches in designing effective and efficient drug delivering system. It has been demonstrated that the applications of nanomaterials, such as carbon nanotubes, silica nanowires and gold nanoparticles have increased the stability and durability of the drugs [5]. The enhanced activity of nanoparticles as drug delivery systems has broadened the application of nanotechnology in antitumor drug design [5].

There is also an increased scientific interest in exploring the potential of layered nanomaterials in slow and controlled release drug delivery applications. In this backdrop, layered double hydroxides (LDHs) which are a class of clays, composed of brucite like nanolayers with a thickness of $0.48 \mathrm{~nm}$, and interlayer charge balancing anions have gained a specific attention. Interestingly, these interlayer anions are able to undergo ion exchange reactions thus, allowing to engineer a range of layered materials with preferred properties. For example, Li et al. intercalated Fenbufen as anti- inflammatory drug, into the LDH matrix with a coating of enteric polymers to be used in the digestive tract as a slow release drug formulation [6]. Samindra and Kottegoda have reported the successful intercalation of natural antibacterial curcumin , into Mg: $\mathrm{Al}$ (2:1 ratio) $\mathrm{LDH}$ and demonstrated its slow release behavior [7]. Latip et al. intercalated anionic ciprofloxacin, which is a broad spectrum antibiotic compound in therapeutic purpose, into $\mathrm{Zn} \mathrm{Al-} \mathrm{LDH} \mathrm{and}$ the sustained release and the enhanced toxicity effect were studied [8]. Furthermore, there had been several successful attempts of using LDHs in order to intercalate antibacterial compounds especially in approaches of pharmaceutical, cosmetic and agricultural industries. Bugatti et al. has intercalated 2,4-dichlorobenzoate, benzoate and para- hydroxybenzoate which are commonly used as antimicrobial agents in food packaging purposes, into the $\mathrm{Zn} \mathrm{Al-} \mathrm{LDH} \mathrm{via} \mathrm{anion} \mathrm{exchange} \mathrm{reac-}$ tion [9]. Antibacterial activity and enhanced release behavior of these LDHs have been studied and confirmed.

In this study, attempts are made to encapsulate citrate anions into an LDH matrix and to study its potential as a slow release antifungal agent in skin topical applications.

\section{Results and discussion}

\section{Characterisation of citrate-LDH}

PXRD technique was used tounderstand the successful intercalation of citrate ions into the LDHs (Fig. 1).

The PXRD pattern of pure nitrate - LDH agrees well with that reported in literature [6]. Based on a hexagonal unit cell the PXRD peaks were indexed resulting in unit cell parameters of $c=2.52 \mathrm{~nm}$ and $a=0.27 \mathrm{~nm}$. Considering the basal reflections an inter-layer spacing of $0.87 \mathrm{~nm}$ leading to a gallery height of $0.39 \mathrm{~nm}$ is suggested and the observed value agrees with previous results $(0.88 \mathrm{~nm})$ [6]. This gallery height suggests a vertical arrangement of nitrate anions within the layers, considering the size of nitrate ions as $0.4 \mathrm{~nm}$ [10].

When compared with the standard nitrate - LDH, the PXRD pattern of citrate-LDH demonstrates a lower wave number shift for the basal peaks (003 and 006), while there is no noticeable change in the non-basal reflections. This suggests that there is an increase in the interlayer spacing after intercalation of citrate thus the $c$ parameter of the unit cell while there is no effect to the a parameter thus suggesting a constant inter-metallic distance. The observed interlayer spacing for citrate$\mathrm{LDH}$ is $1.5 \mathrm{~nm}$. Given that the thickness of the brucitelike layer of $\mathrm{LDH}$ is $0.48 \mathrm{~nm}$, the gallery height of $1.02 \mathrm{~nm}$ is observed. Considering the size of citrate ion as $0.72 \mathrm{~nm}$ [11], vertical monolayer arrangement can be suggested within the interlayer region (Fig. 2).

The absence of any peaks arising from nitrate- LDH confirms that there is no nitrate intercalation in citrate$\mathrm{LDH}$ due to anionic competition during the synthesis. The observation could be explained referring to the preferences of anionic valency during formation of LDHs. Generally, higher valent anions are preferred over lower valent anions due to the lower steric hindrance that arise within the interlayer spaces. In addition citrate ions create a very strong hydrogen bond network within the interlayer region due to the presence of $\mathrm{OH}$ and carboxylate groups.

The nature of the bonding environment of citrate ions within the layers were studied referring to shifts of hydroxyl and carbonyl peaks of citrate ions in FTIR spectra (Fig. 3).

Broad and intense peak is observed at $3485 \mathrm{~cm}^{-1}$ for pure citric acid which is assigned to the hydroxyl stretching frequency. Sharp intense peak at $1686 \mathrm{~cm}^{-1}$ for pure citric acid is due to the carboxyl stretching modes. For nitrate-LDH, the broad peak at $3390 \mathrm{~cm}^{-1}$ is due to the hydroxyl stretching modes of the layered hydroxyl groups and the peak at $1359 \mathrm{~cm}^{-1}$ is assigned for the nitrate group stretching frequency which present within the layers. Intercalation leads to the formation of strong $\mathrm{H}$ bonding network. Therefore, peak broadening can be observed in nitrate-LDH than in pure citric acid.

Intense broad peak is observed at $3354 \mathrm{~cm}^{-1}$ for citrate-LDH corresponding to the hydroxyl and water stretching frequencies. These lower wave number shift from the pure citrate ions and nitrate-LDH suggest the increased electron density around the functional groups. The strong $\mathrm{H}$-bonding network is responsible for the improved electron density around $\mathrm{OH}$ groups. When 


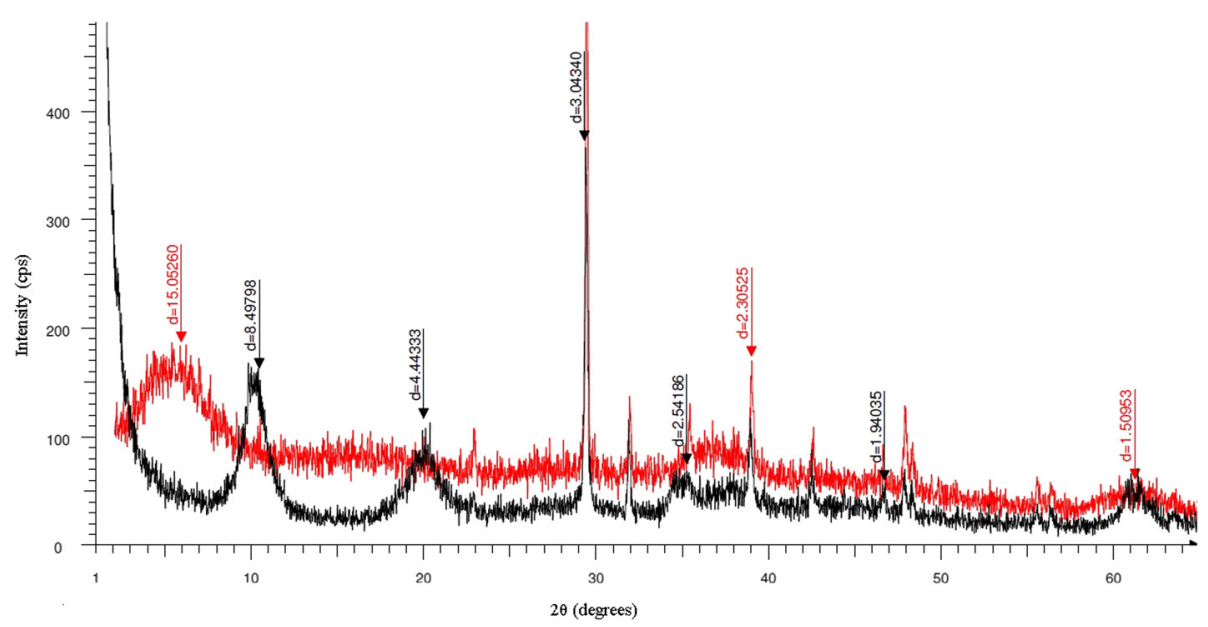

Fig. 1 PXRD patterns of nitrate - LDH (black) citrate - LDH (red) PXRD patterns were recorded on the dried $\left(100^{\circ} \mathrm{C}\right)$ white powder obtained by insitu co-precipitation reactions

compared with the nitrate-LDH, for citrate-LDH anions has the ability to form stronger $\mathrm{H}$-bonding network than the nitrate groups due to the presence of larger number carboxylate and hydroxyl groups. This leads to the peak broadening in citrate-LDH spectra. Sharp peaks observed at $1567 \mathrm{~cm}^{-1}$ and $1386 \mathrm{~cm}^{-1}$ for citrate-LDH correspond to the asymmetric and symmetric stretching modes of the carboxylate groups. The stretching vibrations of the carboxylate group of pure citric acid at $1686 \mathrm{~cm}^{-1}$ has been shifted to $1350-1600 \mathrm{~cm}^{-1}$ after the intercalation into layers. That is due to the stronger intermolecular interactions such as $\mathrm{H}$ - bonding and Van Der - Waals interactions, experienced by the intercalated citrate anions within the matrix. Those interactions stabilize the system and decrease the vibration modes of the molecule.

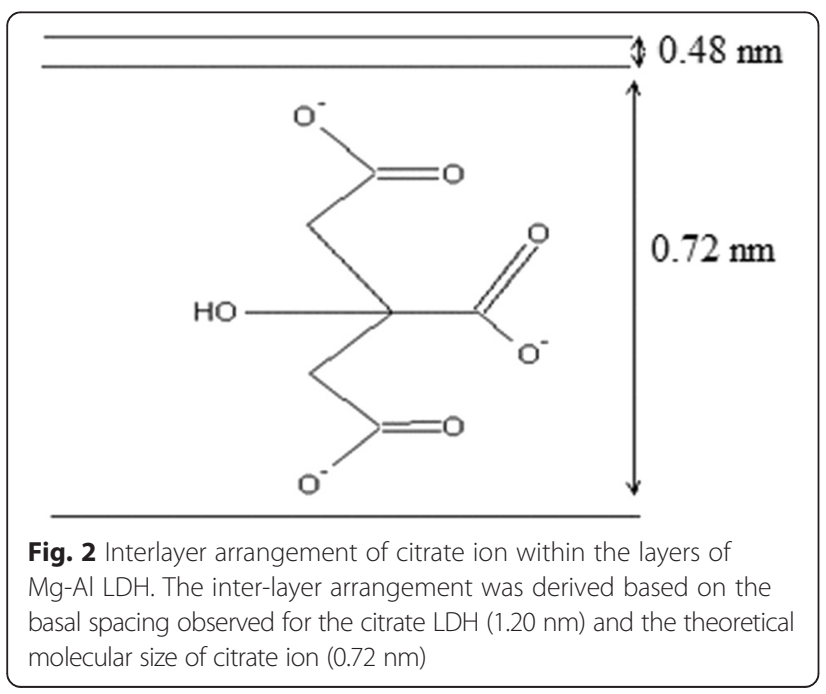

Nitrate stretching peak at $1359 \mathrm{~cm}^{-1}$ is absent in citrateLDH confirming that the formed LDH does not contain any intercalated nitrate ions. The FTIR characterization verifies the successful synthesis of citrate-LDH nanohybrid.

The morphological features of the citrate-LDH was studied using scanning electron microscopic imaging, Fig. 4. As evidenced by the image the composite demonstrated a typical layered structure containing nanometer size thick platelets. The crystallinity of the LDH was low according to the SEM images further corroborating the PXRD data. EDX data suggested a Mg: $\mathrm{Al}$ ratio of 2 as expected.

\section{Release behavior of citrate-LDH and the body lotion at different $\mathrm{pH}$ values}

Release behavior of the citrate-LDH, pure citric acid were compared at different $\mathrm{pH}$ values $(3,4,5)$ while that was repeated for citrate-LDH intercalated body lotion at $\mathrm{pH} 4$ which is the skin $\mathrm{pH}$ under Candida species infections.

As shown in Fig. 5 citrate-LDH shows a gradual release of intercalated citrate ions into the medium over a considerable time period. Within the first eight hours citrate is released in a slow and sustainable manner while a constant releasing pattern is observed afterwards. On the other hand, pure citrate shows a rapid releasing pattern, where all the added citrate ions were released within the first hour. This observation confirms the slow releasing ability of citrate-LDH is a result of the layered matrix of LDH.

Furthermore, the amount of citrate released at $\mathrm{pH} 3$ is higher than that of $\mathrm{pH} 4$ and $\mathrm{pH} 5$. In the acidic medium protons attack to the citrate ions and thereby citrate ions get protonated and released to the medium 


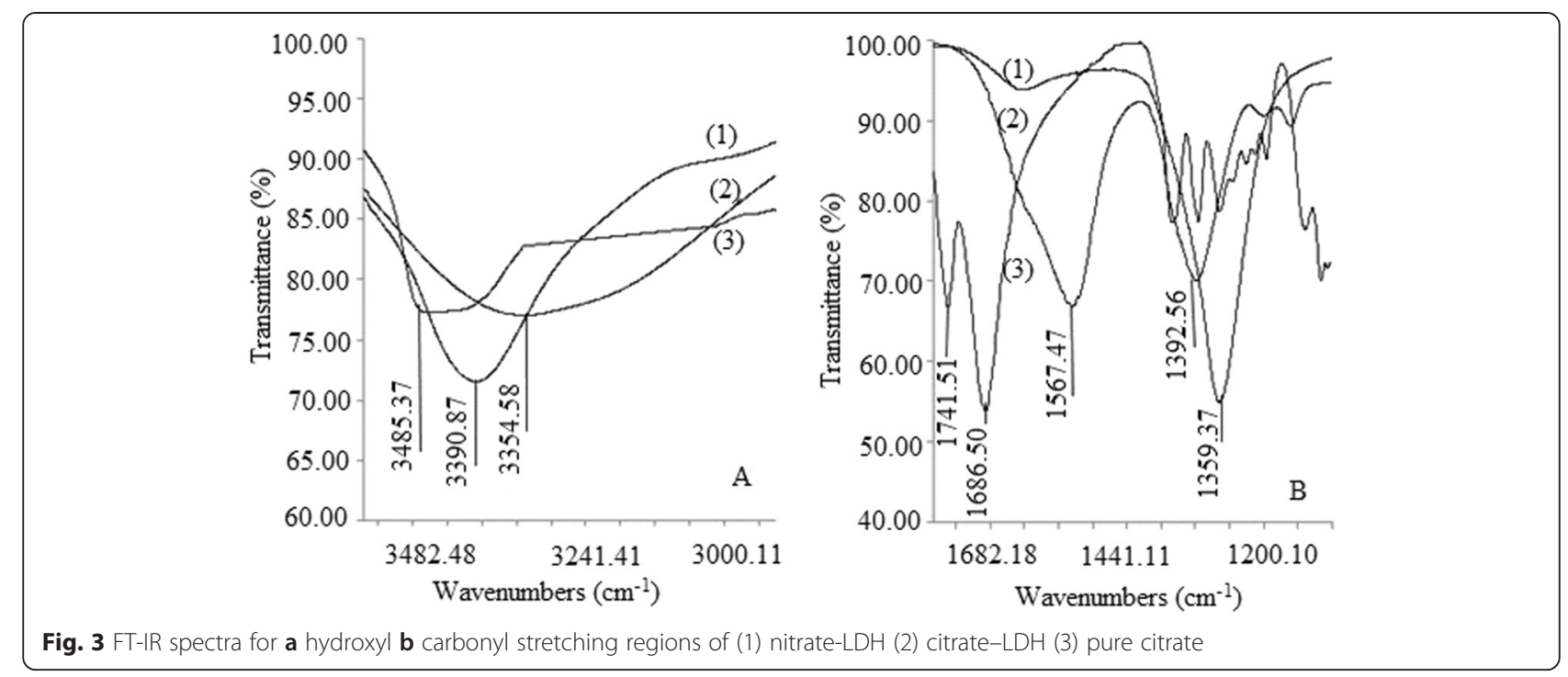

thus leading to higher amount of citrate ions to release from the layered matrix to the medium.

The release behavior of the citrate-LDH incorporated body lotion is compared with that of citrate incorporated body lotion at $\mathrm{pH} 4$ which is closer to the skin $\mathrm{pH}$ under fungal infections (Fig. 6). According to the above result it is confirmed, that the citrate-LDH incorporated lotion also shows a similar slow release pattern of intercalated citrate ions into the medium to the citrate- $\mathrm{LDH}$, over a considerable time period. However, only $40 \%$ of the intercalated citrate is released within the first eight hours. That limitation to the release of citrate may be due to the effect from the lotion matrix. This observation provides evidence of the potential of citrate-LDHs to be used as slow release antifungal formulations in topical dermatological applications.

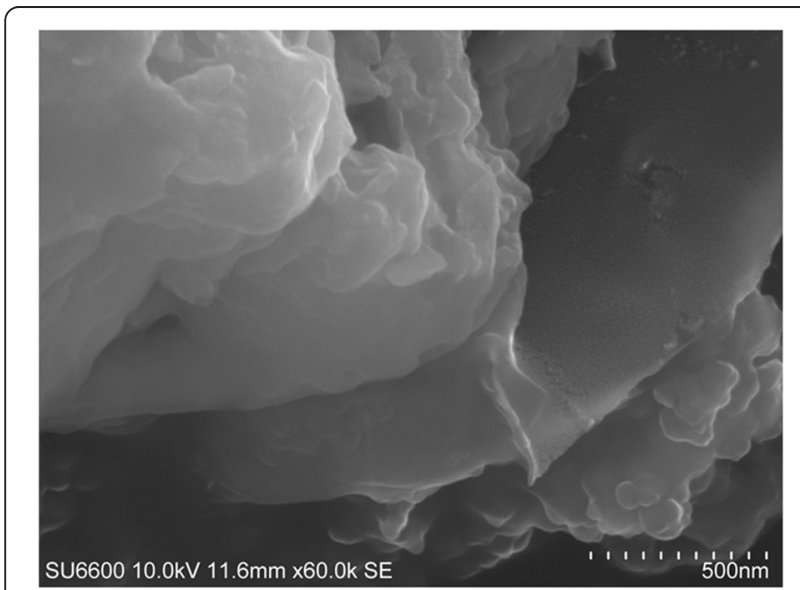

Fig. 4 SEM image of citrate-LDH. The internal morphologial features of the citrate-LDH is demonstrated in this image. The images have been captured in the secondary electron mode with a magnification of 60,000 and an accelerating voltage of $10 \mathrm{k} \mathrm{eV}$

\section{Antimicrobial properties against Candida species}

The antifungal activities of pure citrate, citrate-LDH and citrate-LDH incorporated lotion were tested against the growth of Candida albicans, Candida tropicalis and Candida glabrata. Organic acids are widely used as buffer agents in medical solutions as well as the preservatives in food [12]. In addition to their inhibitory effect on the growth of food spoilage microorganisms, organic acids were shown antibacterial and antifungal activity against various infectious pathogens [13]. These organic acids may have several inhibitory mechanisms. It may, cause the reduction of interior $\mathrm{pH}$ of microbial cells or depression of proton motive force [12]. It may also prevent the production of malic acid, which is an important intermediate in the cellular respiration, thereby reduce the energy production of microorganisms and inhibit their growth [13].

According to the observations in the study; pure citrate, citrate-LDH and citrate-LDH incorporated lotion shows inhibitive activity against all three strains of Candida. When compared with the pure citric acid, incorporated citrate is able to inhibit the growth of fungi for longer time period (Table 1). Since the lotion matrix does not shows any activity against the growth of the fungi, the inhibitive action of the citrate-LDH incorporated lotion is approached only by the incorporated citrate ions.

Although C. albicans and C. glabrata express the similar growth response towards the tested substances, behaviour of the $C$. tropicalis towards the same substances after $48 \mathrm{~h}$ is altered. That type strain of Candida $s p$. may have the ability to adjust to the changes in the growth medium made by the organic acid. Therefore, the inhibitory activity of citric acid and intercalated citrate is efficient towards the growth of C. albicans and C. glabrata but not that efficient towards C. tropicalis. 


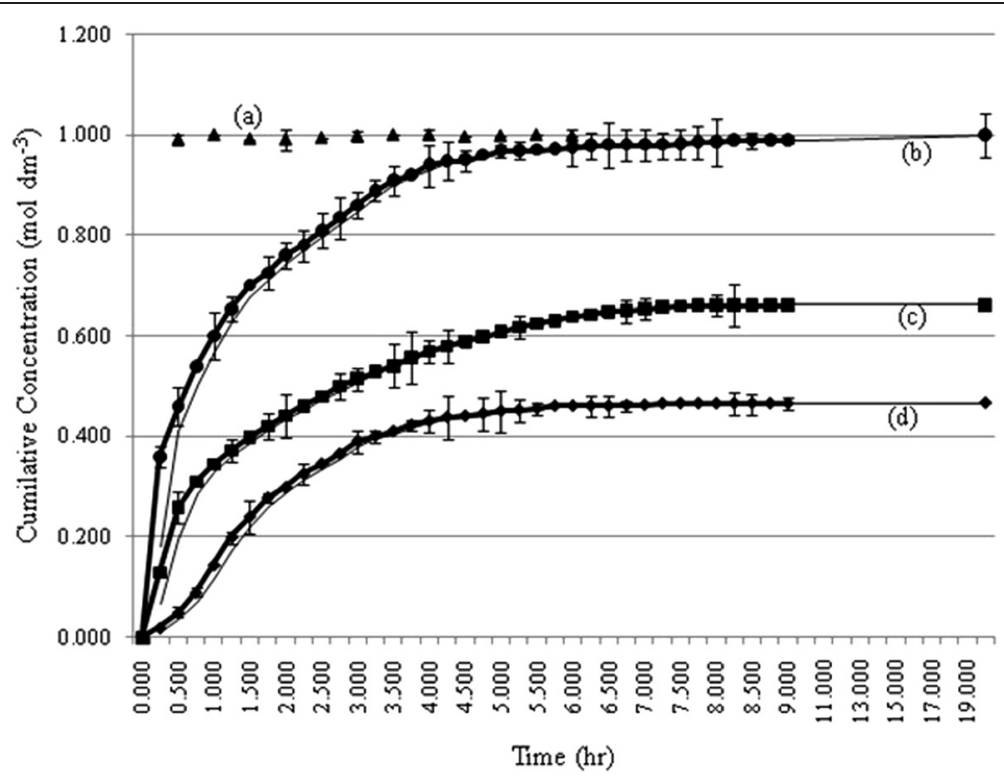

Fig. 5 Release behavior of a citric acid ( $\mathrm{pH} 5)$, citrate-LDH at $\mathrm{pH}$ b 3, c 4 and $\mathbf{d} 5$

\section{Synthesis of $L D H s$}

Nitrate and citrate intercalated Mg-Al-LDHs (Mg:Al 2:1) were prepared. Nitrate intercalated LDH (nitrate=LDH) was prepared as explained by Li et al. [6] and was used as the standard for the comparison purposes. Citrate intercalated LDH (citrate-LDH) was synthesized to explore its potential as a slow release anti-microbial compound in skin formulations.

A solution containing $0.25 \mathrm{~mol}$ of $\mathrm{Mg}\left(\mathrm{NO}_{3}\right)_{2} \cdot 6 \mathrm{H}_{2} \mathrm{O}$ and $0.125 \mathrm{~mol}$ of $\mathrm{Al}\left(\mathrm{NO}_{3}\right)_{3} .9 \mathrm{H}_{2} \mathrm{O}$ in $250.00 \mathrm{~cm}^{3}$ of distilled water was added drop wise to a solution containing $0.25 \mathrm{~mol}$ of $\mathrm{NaNO}_{3}$ in $100.00 \mathrm{~cm}^{3}$ of distilled water, under an inert atmosphere and vigorous stirring condition at $60{ }^{\circ} \mathrm{C}$. The $\mathrm{pH}$ of the solution was maintained at 10 throughout the experiment using a solution of
$2.0 \mathrm{~mol} \mathrm{dm}{ }^{-3} \mathrm{NaOH}$. Then, the solution was aged at $60{ }^{\circ} \mathrm{C}$ for $14 \mathrm{~h}$ and the precipitate obtained, was separated by filtration. Then, the precipitate was washed thoroughly with distilled water until excess $\mathrm{NaOH}$ get removed and dried at $80{ }^{\circ} \mathrm{C}$ for $18 \mathrm{~h}$ resulting in nitrate intercalated LDH.

Citrate intercalated LDH was prepared using a similar procedure as explained for nitrate-LDH. Instead of 2.5 moldm ${ }^{-3}$ of $\mathrm{NaNO}_{3}, 2.55 \mathrm{~mol} \mathrm{dm}{ }^{-3}$ of tri sodium citrate solution was used as the anion to be intercalated.

\section{Experimental}

All chemicals and reagents used for the experiments were of analytical grade and distilled water was used for the experimental work.

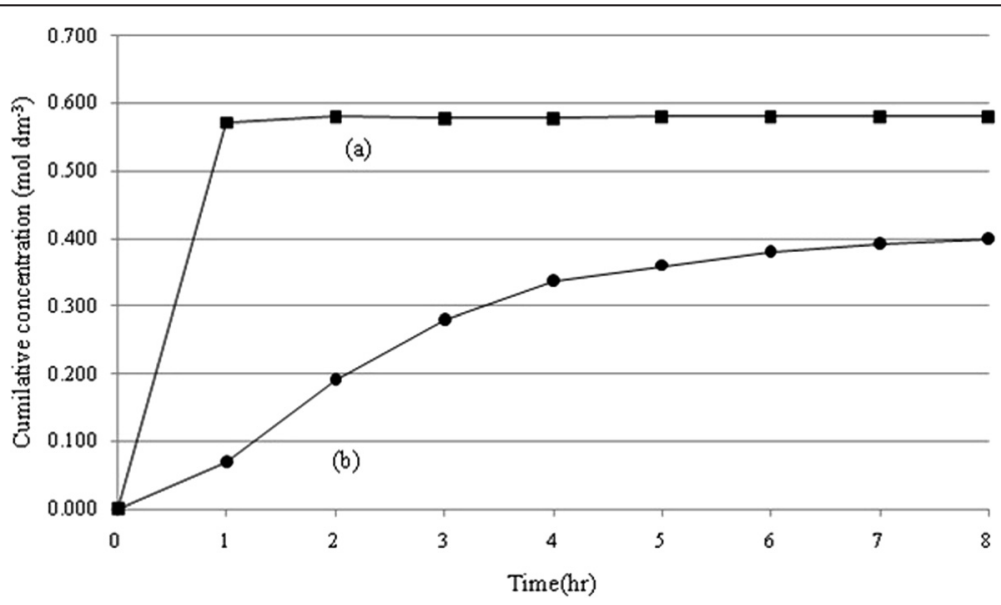

Fig. 6 Release behavior of the a citrate ion incorporated body lotion b citrate-LDH incorporated body lotion 
Table 1 Inhibitory activity of test compounds against C. albicans, C. glabrata and C. tropicalis

\begin{tabular}{|c|c|c|c|c|c|c|}
\hline \multirow[t]{3}{*}{ Substances tested } & \multicolumn{6}{|c|}{ Visually inspected growth } \\
\hline & \multicolumn{2}{|c|}{ Candida albicans } & \multicolumn{2}{|c|}{ Candida glabrata } & \multicolumn{2}{|c|}{ Candida tropicalis } \\
\hline & After $24 \mathrm{~h}$ & After $48 \mathrm{~h}$ & After $24 \mathrm{~h}$ & After $48 \mathrm{~h}$ & After $24 \mathrm{~h}$ & After $48 \mathrm{~h}$ \\
\hline Fluconazole & 0 & 0 & 0 & 0 & 0 & +1 \\
\hline Pure citric acid & 0 & +2 & 0 & +2 & 0 & +2 \\
\hline Citrate-LDH & 0 & 0 & 0 & 0 & 0 & +1 \\
\hline Body lotion matrix & +1 & +2 & +2 & +2 & +1 & +2 \\
\hline Citrate-LDH incorporated lotion & 0 & 0 & 0 & 0 & 0 & +1 \\
\hline
\end{tabular}

The study of slow release antifungal activity is based on visual observation of the fungal growth in a Muller Hinton culture media and the criteria used is given below

+2 : Growth is equals to that of the negative control

+1 : Growth approximately $50 \%$ that of the negative control

0 : No visible growth at all

\section{Incorporation of citrate anions and citrate $=L D H$ into $a$ body} lotion formulation

Citrate anion and citrate-LDH were incorporated into a body lotion formulation as explained below.

A mixture containing distilled water and glycerin (2:1 ratio) was heated for $20 \mathrm{~min}$ and added to a mixture containing unrefined pure cocoa butter and emulsifier (1:2 ratio), while continues homogenizing. Then, the mixture was cooled down to room temperature and $1.00 \mathrm{~g}$ of tri sodium citrate was added and homogenized for $1 \mathrm{~h}$. A similar procedure was followed to introduce citrate-LDH in to the body lotion formulation.

\section{Release behavior studies}

Release behavior studies of the citrate-LDH (1.00 g of citric acid), and pure citric acid (1.00 g) were performed using a dialysis membrane bag containing a magnetic stirrer, dipped in a receptor compartment containing $20.00 \mathrm{~mL}$ of

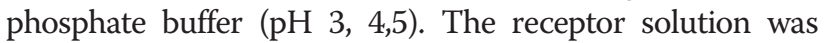
stirred at room temperature and at suitable time intervals the releasing medium $(5.00 \mathrm{~mL})$ was collected. The amount of released citrate was measured and quantified by UVVisible spectrophotometer. Release behavior studies of the pure citrate (equivalent weight is $0.632 \mathrm{~g}$ ) and citrate-LDH incorporated lotion were carried out using the same method with the receptor solution at $\mathrm{pH} 4$.

\section{Determination of antifungal activity}

Preparation of sample The antifungal activity against three Candida species (Candida albicans (ATCC 90028), Candida tropicalis (ATCC 13803), and Candida glabrata (ATCC 90030)) of the nanohybrid formulation was compared with that against citrate and fluconazole, one of the common topical antifungal administration.

Solutions of $0.25 \mathrm{~g} \mathrm{dm}^{-3}$ citric acid, citrate-LDH and fluconazole were prepared using normal saline and the solutions were autoclaved before the experiments.

Preparation of agar medium for pour plate method Mueller- Hinton agar (Oxoid, England) medium was prepared and after autoclave the media was left to semi cool in a sterilized environment [14]. Pre prepared samples $(2.00 \mathrm{~mL}$ of each) were placed in separate sterile glass plates and $18.00 \mathrm{~mL}$ of prepared agar medium was then poured into plates and swirl clockwise and anticlockwise and allowed to solidify.

Preparation of inoculum Three of type strains of Candida species (Candida albicans (ATCC 90028), Candida tropicalis (ATCC 13803), and Candida glabrata (ATCC 90030)) were used to study the antifungal activity. Young (24 h old) and pure cultures of type strains were inoculated in Muller - Hinton agar (Oxoid, England) plates and incubated at $37{ }^{\circ} \mathrm{C}$ for $24 \mathrm{~h}$ [14]. Then, inoculums were prepared by dissolving loop-full of each species in sterile normal saline and the turbidity of suspension was adjusted to $0.5 \mathrm{McF}$ arland standard.

Inoculation and incubation of the medium Inoculation and incubation of the medium was carried out as explained by Nadeem and co-workers [15]. Each prepared inoculums of C. albicans, C. tropicalis and $C$. glabrata were then separately spotted $(40.00 \mu \mathrm{L})$ on the prepared culture plates. All the experiments were carried out in triplicates. Fluconazole was used as the standard laboratory control while sterile distilled water was used as a negative control for the experiment. The inoculated plates were incubated at $37{ }^{\circ} \mathrm{C}$ aerobically in an incubator and fungal growth in each plate was observed after $24 \mathrm{~h}$ and $48 \mathrm{~h}$.

\section{Characterization}

Powder X-Ray Diffraction (PXRD) characterization was carried out to identify the crystalline phases in the synthesized LDHs. Brucker D8 focus X-ray powder diffraction meter was used to analyze the powdered LDHs using $\mathrm{Cu} K \alpha$ radiation (wave length $-1.540 \AA$ ) over $2 \theta$ angle, of $2^{0}$ to $70^{\circ}$ with a step size of $0.02^{\circ}$.

Fourier Transform Infra-Red (FTIR) spectroscopy technique was used to identify functional groups in the 
synthesized materials. Nicolet IS 10 instrument was used to analyze the powdered sample using diffuse reflection mode in the range from $600 \mathrm{~cm}^{-1}$ to $4000 \mathrm{~cm}^{-1}$. The sample was mixed with potassium bromide in 1:100 ratio and then the mixture was ground to a fine powder. Then, a disc having an even surface was prepared by compressing the powdered sample.

Thermo Gravimetric Analysis (TGA) was used to study the weight loss of the material as the function of the temperature allowing to understand the thermal stability of the synthesized materials. SDTQ 600 thermo gravimetric analyzer was used in this study. The sample $(10 \mathrm{mg})$ was heated at a rate of $10^{\circ} \mathrm{C}$ per minute in a nitrogen atmosphere in a temperature range of $30-1000{ }^{\circ} \mathrm{C}$. Q series 600 software was used to this analysis.

UV- 2602 Labomed, Inc. single beam scanning spectrophotometer was used for citric acid release studies as a function of time at different $\mathrm{pH}$ values.

Morphological studies of the citrate LDH was carried out using scanning electron microscopy (SEM) SU6600, in secondary electron mode. Energy dispersive X-ray analysis (EDX) was carried out using the EDX detector attached to the SU6600 SEM.

\section{Conclusion}

Mg-Al-citrate-LDH has been successfully synthesized and introduced into a body cream formulation containing cocoa-butter. The resulting nanohybrid body lotion demonstrated slow release characteristics for citrate anion at acidic $\mathrm{pH}$ values. Improved and prolonged activity of the novel nanohybrid has been proved against Candida albicans and Candida glabrata compared to fluconazole, one of the common topical antifungal drug.

\section{Competing interests}

The authors declare that they have no competing interests.

\section{Authors' contributions}

JP carried out the bench studies, characterized and analyzed the results, and drafted the manuscript. NK designed the project, supervised, guided the overall project and finalized the manuscript. MW designed and guided the anti-fungal studies. All authors read and approved the final manuscript.

\section{Authors' information}

NK is a senior research scientist attached to Sri Lanka Institute of Nanotechnology and a senior lecturer at the Department of Chemistry, University of Sri Jayewardenepura, Sri Lanka. She obtained her Ph. D. in Materials Chemistry from the University of Cambridge, UK. Her current research work spans over a wide spectrum of areas; nanotechnology applications in agriculture, nano nutraceuticals and cosmoceuticals, nanomaterials for water purification, rubber nanocomposites, and synthesis of nanomaterials from natural/mineral resources.

$J P$ is a temporary lecturer attached to the Department of Chemistry, University of Sri Jayewardenepura, Sri Lanka. She obtained her BSc special degree in Chemistry from University of Sri Jayewardenepura in 2015. MW is a senior lecturer attached to the Department of Microbiology, Faculty of Medical Sciences, University of Sri Jayewardenepura, Sri Lanka. She obtained her $\mathrm{PhD}$ in molecular medicine in 2011 from the University of Otago, New Zealand. Her current research interests are antimicrobial resistance, bio-films studies and pathogenesis of medically important microorganisms.

\section{Acknowledgements}

Authors wish to extend their acknowledgements to Departments of Chemistry of University of Sri Jayewardenepura for the financial support and to Sri Lanka Institute of Nanotechnology for providing analysis facilities.

\section{Author details}

${ }^{1}$ Department of Chemistry, Faculty of Applied Science, University of Sri Jayewardenepura, Nugegoda, Sri Lanka. ${ }^{2}$ Sri Lanka Institute of Nanotechnology, Center for Excellence in Nanotechnology, Nanotechnology and Science Park, Pitipana, Homagama, Sri Lanka. ${ }^{3}$ Department of Microbiology, Faculty of Medical Science, University of Sri Jayewardenepura, Nugegoda, Sri Lanka.

Received: 13 February 2015 Accepted: 14 May 2015

Published online: 21 May 2015

\section{References}

1. Havlickova B, Czaika VA, Friedrich M. Epidemiological trends in skin mycoses worldwide. Mycoses. 2008;51(s4):2-15.

2. Yang Y-L. Virulence factors of Candida species. J Microbiol Immunol Infect. 2003;36(4):223-8.

3. Del Hoyo C. Layered double hydroxides and human health: an overview. Appl Clay Sci. 2007;36:103-21

4. Choy J, Choi S, Oh J, Park T. Clay minerals and layered double hydroxides for novel biological applications. Appl Clay Sci. 2007;36(1-3):122-32.

5. Reddy PD, Swarnalatha D. Recent advances in novel drug delivery systems. International Journal of PharmTech Research. 2010;2(3):2025-7.

6. Li B, He J, Evans D, Duan X. Inorganic layered double hydroxides as a drug delivery system intercalation and in vitro release of Fenbufen. Appl Clay Sci. 2004;27:199-207.

7. Samindra KMS, Kottegoda N. Encapsulation of curcumin into layered double hydroxides. Nanotech Rev. 2014;3(6):579-89.

8. Latip AFA, Hussein MZ, Stanslas J, Wong CC, Adnan R. Release behavior and toxicity profiles towards A549 cell lines of ciprofloxacin from its layered zinc hydroxide intercalation compound. Chem Cent J. 2013;7(1):119.

9. Bugatti V, Costantino U, Gorrasi G, Nocchetti M, Tammaro L, Vittoria V. Nano-hybrids incorporation into poly ( $\varepsilon$ - caprolactone) for multifunctional applications: Mechanical and barrier properties. Eur Polym J. 2010;46(3):418-27.

10. Choy J-H, Jung J-S, Oh J-M, Park M, Jeong J, Kang Y-K, et al. Layered double hydroxide as an efficient drug reservoir for folate derivatives. Biomaterials. 2004;25(15):3059-64.

11. Tronto J, dos Reis MJ, Silvério F, Balbo VR, Marchetti JM, Valim JB. In vitro release of citrate anions intercalated in magnesium aluminium layered double hydroxides. J Phys Chem Solid. 2004;65(2):475-80.

12. Phillips $C$. The effect of citric acid, lactic acid, sodium citrate and sodium lactate, alone and in combination with nisin, on the growth of Arcobacter butzleri. Lett Appl Microbiol. 1999;29(6):424-8.

13. Shokri H. Evaluation of inhibitory effects of citric and tartaric acids and their combination on the growth of Trichophyton mentagrophytes, Aspergillus fumigatus, Candida albicans and Malassezia furfur. World I Zool. 2011;6(1):12-5.

14. Jagessar R, Mohamed A, Gomes G. Antibacterial and antifungal activity of leaf extracts of Luffa operculata vs Peltophorum pterocarpum against Candida albicans, Staphylococcus aureus and Escherichia coli. Nat Sci. 2007;5(4):81-93.

15. Nadeem SG, Shafiq A, Hakim ST, Anjum Y, Kazm SU. Effect of growth media, $\mathrm{pH}$ and temperature on yeast to hyphal transition in Candida albicans. Open J Med Microbiol. 2013;3(3):185-92. 